\title{
Subacute Effects of Vaccination with a Messenger RNA- based Vaccine Against Coronavirus Disease 2019 on Elderly Japanese Patients with Cardiac Disorder
}

\section{Ryuichi Tamimoto}

Tokai University School of Medicine

Toshiharu Fujii

Tokai University School of Medicine

Hirofumi Nagamatsu

Tokai University School of Medicine

Tsutomu Murakami

Tokai University School of Medicine

Koji Miyazaki

Tokai University Hachiouji Hospital

Shinya Goto

Tokai University School of Medicine

Yasunori Cho

Tokai University School of Medicine

Hidezo Mori ( $\nabla$ hidezomori@hotmail.com )

Tokai University School of Medicine

\section{Research Article}

Keywords: coronavirus disease 2019, vaccine, death, brain natriuretic peptide, heart failure

Posted Date: February 28th, 2022

DOI: https://doi.org/10.21203/rs.3.rs-1391307/v1

License: (c) (i) This work is licensed under a Creative Commons Attribution 4.0 International License. Read Full License 


\section{Abstract}

The present observational study assessed the occurrence of adverse cardiac events following vaccination of patients $(n=100)$ with an mRNA-based vaccine against coronavirus disease 2019 (COVID-19). Death and deterioration of heart failure were evaluated as side effects. The status of heart failure was assessed using the brain natriuretic peptide (BNP) ratio, for which the BNP or NT-pro BNP values were divided by their upper normal limits (UNL); values (i.e., post-BNP levels minus pre-BNP levels) $\geq 10$-fold higher than the UNL denoted significant increment. Here we show that vaccination with an mRNA-based vaccine against COVID-19 was associated with a high risk of death or decompensated heart failure for elderly patients with a BNP ratio $\geq 4$-fold higher than the UNL. Six and 15 patients expired and exhibited significant increment of the BNP ratio, respectively; these patients had a pre-BNP ratio $\geq 4$-fold higher than the UNL. Forty-five patients with a pre-BNP ratio < 4-fold higher than the UNL did not expire or show significant increase in the BNP ratio. The pre-BNP ratio was a reliable parameter for predicting cardiac deterioration after vaccination. For the booster or initial vaccination against COVID-19, the BNP ratio may be useful in assessing the cardiac status of elderly patients.

\section{Introduction}

It is established that vaccination using an mRNA-based vaccine against coronavirus disease 2019 (COVID-19) is effective in preventing the spread of infection. Moreover, acute myocarditis in young individuals has been reported as a side effect of this type of vaccination. ${ }^{1-4}$

However, it is currently unknown whether vaccination with an mRNA-based vaccine against COVID-19 has serious side effects on elderly patients with cardiac diseases in the subacute phase. Therefore, the objective of this study was to investigate the occurrence of adverse cardiac events 1-8 months following vaccination with an mRNA-based vaccine against COVID-19 (COMIRNATY® from BIONTECH and Pfizer Pharmaceutical Co. Ltd., Tokyo Japan).

\section{Results}

Baseline characteristics are summarized in Table 1. Six patients expired during a median follow-up of 152.5 days (interquartile range: 20-222 days) after the first vaccination. The pre- and post-BNP ratios of patients who expired and survived are demonstrated in Fig. 1. Both pre- and post-BNP ratios were higher in patients who expired (23.9 [12.6-43.6] and 144 [33.8-280], respectively) than in those who survived (4.2 [1.4-9.5] and 5.2 [1.8-16.1], respectively). The degree of increase after vaccination (i.e., post-BNP ratio minus pre-BNP ratio) was significantly high ( $\geq 10$-fold higher than their $\mathrm{UNL}$ ) in five patients who expired and 15 patients who survived. Death and/or remarkable increase in the BNP ratio (i.e., $\geq 10$-fold higher than the UNL) were observed only in patients with a pre-BNP ratio $\geq 4$-fold higher than the UNL (21/55, $38.2 \%$ ) (Table 2). In contrast, 45 patients with a pre-BNP ratio < 4-fold higher than the UNL in the pre-vaccination period did not expire or show remarkable increase in the BNP ratio $(0 / 45,0 \%)($ Table 2$)$. Hence, the pre-BNP ratio was a reliable parameter for predicting cardiac deterioration 1-8 months after vaccination (crude HR: 1.02 [95\% $\mathrm{Cl}: 1.01-1.03 ; P<$ 0.01]); adjusted HR: 1.02 [95\% Cl: 1.00-1.03; $P=0.03$ ]) (Table 3). 
Table 1

Baseline characteristics $(n=100)$

\begin{tabular}{|c|c|c|c|}
\hline Characteristic & Baseline & Post vaccination & $P$ value \\
\hline Age, years & $84(78-89.5)$ & NA & NA \\
\hline Males, n & $45(45 \%)$ & NA & NA \\
\hline Height, cm & $155.5(145.5-163.5)$ & NA & NA \\
\hline Weight, kg & $51(42.5-60)$ & NA & NA \\
\hline BMI & $21.4(19.0-24.3)$ & NA & NA \\
\hline Hypertension, $\mathrm{n}$ & $64(64 \%)$ & NA & NA \\
\hline Dyslipidemia, $\mathrm{n}$ & $54(54 \%)$ & NA & NA \\
\hline Diabetes mellitus, $\mathrm{n}$ & $21(21 \%)$ & NA & NA \\
\hline Prior myocardial infarction, $\mathrm{n}$ & $16(16 \%)$ & NA & NA \\
\hline Anticoagulation, $\mathrm{n}$ & $35(35 \%)$ & NA & NA \\
\hline Warfarin & $17(17 \%)$ & NA & NA \\
\hline DOAC & $18(18 \%)$ & NA & NA \\
\hline Serum creatinine, $\mathrm{mg} / \mathrm{dL}$ & $0.9(0.7-1.1)$ & $0.9(0.7-1.1)$ & 0.26 \\
\hline Urea nitrogen, mg/mL & $20.6(15.9-26.0)$ & $21.6(16.6-26.1)$ & 0.33 \\
\hline Missing, $n$ & 4 & 3 & \\
\hline AST, IU/L & $21(18-25.5)$ & $22(18-25)$ & 0.22 \\
\hline Missing, $\mathrm{n}$ & 4 & 3 & \\
\hline ALT, IU/L & $15(12-21)$ & $16(11-22)$ & 0.92 \\
\hline Missing, $\mathrm{n}$ & 4 & 3 & \\
\hline $\mathrm{HbA} 1 \mathrm{c}, \%$ & $5.8(5.4-6.2)$ & $5.8(5.4-6.1)$ & 0.02 \\
\hline Missing, $\mathrm{n}$ & 16 & 19 & \\
\hline Troponin T, ng/mL & $0.03(0.01-0.07)$ & $0.03(0.02-0.07)$ & 0.55 \\
\hline Missing, $\mathrm{n}$ & 32 & 24 & \\
\hline CRP, mg/dL & $0.09(0.04-0.26)$ & $0.09(0.05-0.28)$ & 0.02 \\
\hline Missing, $\mathrm{n}$ & 28 & 15 & \\
\hline Abnormal Q wave, $\mathrm{n}$ & $16(16 \%)$ & $12(22 \%)$ & \\
\hline \multicolumn{4}{|c|}{$\begin{array}{l}\text { ALT, alanine aminotransferase; AST, aspartate aminotransferase; BMI, body mass index; BNP, brain natriuretic } \\
\text { peptide; CRP, C-reactive protein; DOAC, direct oral anticoagulant; } \mathrm{HbA1c} \text {, hemoglobin A1c; UNL, upper normal limit. }\end{array}$} \\
\hline \multicolumn{4}{|c|}{${ }^{\dagger}$ Average value prior to first vaccine / upper limit within normal range. } \\
\hline
\end{tabular}




\begin{tabular}{|c|c|c|c|}
\hline Characteristic & Baseline & Post vaccination & $P$ value \\
\hline Missing, $n$ & 2 & 45 & \\
\hline Observation period, days $\S$ & $152.5(20-222)$ & & NA \\
\hline BNP ratio & $4.5(1.5-13.6)^{\dagger}$ & $5.9(1.8-19.5)^{\ddagger}$ & $<0.01$ \\
\hline$<4$-fold higher than the UNL, $n$ & $45(45 \%)$ & $41(40 \%)$ & $<0.01$ \\
\hline$\geq 4,<10$-fold higher than the UNL, $n$ & $27(27 \%)$ & $22(22 \%)$ & \\
\hline$\geq 10$-fold higher than the UNL, $n$ & $28(28 \%)$ & $37(37 \%)$ & \\
\hline Death, n & NA & $6(6 \%)$ & NA \\
\hline \multicolumn{4}{|c|}{$\begin{array}{l}\text { ALT, alanine aminotransferase; AST, aspartate aminotransferase; BMI, body mass index; BNP, brain natriuretic } \\
\text { peptide; CRP, C-reactive protein; DOAC, direct oral anticoagulant; HbA1c, hemoglobin A1c; UNL, upper normal limit. }\end{array}$} \\
\hline \multicolumn{4}{|c|}{${ }^{\dagger}$ Average value prior to first vaccine / upper limit within normal range. } \\
\hline \multicolumn{4}{|c|}{ ‡ Peak value after second vaccine / upper limit within normal range. } \\
\hline$\S$ Duration between the day of first v & nd that of death & ination. & \\
\hline
\end{tabular}

Table 2

Proportions of combined outcome according to the pre-brain natriuretic peptide (pre-BNP) ratio

\begin{tabular}{|c|c|c|c|}
\hline & & \multicolumn{2}{|c|}{ Pre-BNP ratio } \\
\hline & & $<4$ & $\geq 4$ \\
\hline \multirow[t]{2}{*}{ Combined outcome, $n$} & None & 45 (100\%) & 34 (61.8\%) \\
\hline & Presence & 0 & 21 (38.2\%) \\
\hline
\end{tabular}

Table 3

Hazard ratio (HR) for the combined outcome

\begin{tabular}{|lll|}
\hline & Crude HR & $P$ value \\
\hline Pre-BNP ratio & $1.02(1.01-1.03)$ & 0.01 \\
\hline & Adjusted HR & \\
\hline Pre-BNP ratio & $1.02(1.00-1.03)$ & 0.03 \\
\hline Age & $1.07(0.99-1.16)$ & 0.10 \\
\hline Males & $1.63(0.55-4.88)$ & 0.38 \\
\hline BMI & $0.98(0.83-1.15)$ & 0.80 \\
\hline Serum creatinine & $0.26(0.05-1.28)$ & 0.10 \\
\hline Anticoagulation therapy & $5.05(1.54-16.6)$ & 0.01 \\
\hline BMl, body mass index; BNP, brain natriuretic peptide. \\
\hline
\end{tabular}


Six patients expired 47-156 days after the first vaccination. Their pre-BNP ratios ranged 4.8-145.8-fold higher than the UNL (605-18,225 pg/mL in NT-pro BNP) (Fig. 1C). Five of the six patients with a pre-BNP ratio ranging 12.6-145.8-fold higher than the UNL (1,575-18,225 pg/mL in NT-pro BNP) expired due to deterioration of congestive heart failure; a remarkable increase in the BNP ratio (21.2-242.4-fold higher than the UNL) was observed in these patients. The remaining patient (age: 89 years), with the lowest pre-BNP ratio (4.8-fold higher than the UNL) expired due to malnutrition caused by severe back pain which developed after the vaccination. In this patient, we could not determine the BNP ratio after the vaccination. Echocardiographic examination, performed during both the pre- and postvaccination periods, revealed markedly deteriorated left ventricular wall motion after vaccination in four of the six patients who expired. The six patients who expired did not have newly developed abnormal Q waves. A distinct increase in CRP levels (i.e., $>2.0 \mathrm{mg} / \mathrm{dL}$ ) was observed in one of those patients.

The 15 patients who survived despite a significant increase in the BNP ratio had a relatively low pre-BNP ratio (4.241.3-fold higher than the UNL) and increase in BNP ratio (11.5-60.2-fold higher than the UNL) compared with the six patients who expired. In addition, they did not show changes in the electrocardiogram or the troponin T levels, suggesting newly developed myocardial infarction as described above. Seven of the 15 patients who survived exhibited an increase in CRP (i.e., $>2.0 \mathrm{mg} / \mathrm{dL}$ ) in the post-vaccination period. Their post-BNP ratio gradually decreased 1-4 months after the vaccination; however, four patients sustained this increase in the BNP ratio 4-7 months after vaccination (Fig. 1C). In contrast, all patients who expired showed extreme increment of the BNP ratio, excluding one patient who expired due to malnutrition (Fig. 1D).

Acute side effects, such as anaphylactic hemodynamic deterioration or sustained high fever in the acute phase (i.e., within 1 week) were not observed in this study. Most patients did not show significant changes in other blood parameters (i.e., serum creatinine, blood urea nitrogen, aspartate aminotransferase alanine, and aminotransferase) after vaccination.

\section{Discussion}

This study showed that vaccination against COVID-19 was associated with a high risk of death or decompensated heart failure for Japanese elderly patients with severe cardiac dysfunction and a high pre-BNP ratio $\geq 4$-fold higher than the UNL (500 ng/mL in NT-pro BNP). However, the risk was negligible in patients with a pre-BNP ratio < 4-fold higher than the UNL (Table 2). The incidence of death and/or remarkable increase in the BNP ratio (i.e., $\geq 10$-fold higher than the UNL) in the post-vaccination period was strongly dependent on a pre-BNP ratio $\geq 4$-fold higher that the UNL (500 $\mathrm{ng} / \mathrm{mL}$ in NT-pro BNP in Table 2). Cardiovascular death after the vaccination developed only in patients with a pre-BNP ratio > 12.6-fold higher than the UNL (1,575 in NT-pro BNP). Patients with a pre-BNP ratio < 4-fold higher than the UNL in the pre-vaccination period did not expire or show remarkable increase in the BNP ratio after vaccination. This cardiac deterioration was not accompanied by side effects, such as anaphylactic hemodynamic deterioration or sustained high fever in the acute phase, or myocardial infarction, liver dysfunction, or renal dysfunction (Table 1) both in the acute and subacute phases. An increase in CRP ( $>2 \mathrm{mg} / \mathrm{dL}$ ) was noted in one patient who expired and seven of the surviving 15 patients with a remarkable increase in the BNP ratio ( $\geq 10$-fold higher than the UNL). The most likely explanation for this sustained myocardial involvement in the subacute phase would be autoimmune myocarditis; in other words, antiidiotype antibodies-dependent myocarditis recently proposed by Murphy and Longo. 5,6 The primary antibody induced by vaccination against COVID-19 has antigen-binding domains with a mirror image structure of the spike protein of COVID-19. Hence, it may induce a secondary antibody with a mirror image structure of the antigen-binding domains of the primary antibody. Consequently, the secondary antibodies may be able to bind to the angiotensin-converting enzyme 2 (ACE2) receptor as the spike protein of COVID-19, thereby inducing the release of inflammatory cytokines. This hypothesis may explain our observation that myocardial dysfunction was sustained for several months after 
vaccination and led to the death of patients with severe damage. Nevertheless, massive myocardial necrosis was not noted. The transition of the BNP ratio suggests that myocardial damage appears to be somewhat reversible (Fig. 1C D) in certain cases, but sustained in others.

The BNP and/or NT-pro BNP levels in patients with severe cardiac dysfunction may increase during the natural course of the diseases. Therefore, one may argue that the increase in the BNP ratio after vaccination may be caused by the natural course of the diseases. However, in the previous 4 years, the cardiac death rate every 6 months among patients treated by the corresponding author at home or care facilities was 5.0-7.1\% (seven terms). This was accompanied by a maximum BNP ratio ranging 12.6-280-fold higher than the UNL (median: 49.4; interquartile range: 29.7-103.2) and 1,575-35,000 pg/mL in NT-pro BNP (median: 6,180, interquartile range: 3,710-12,900). Nonetheless, the cardiac death rate in the previous 6 months (May 1 to October 31, 2021) was 12 cases (13.5\%), including the six patients who expired after vaccination with an mRNA-based vaccine against COVID-19. The cardiac death rate among patients treated at home or care facilities who did not receive the vaccination during the same period was six cases (6.7\%).

The present study revealed that assessment of the cardiac status, including the BNP ratio, in elderly patients with severe cardiac dysfunction may be useful for determining the risk associated with the booster or initial vaccination against COVID-19. In patients at high risk, lower-dose vaccination should be considered as an alternative option.

\section{Declarations}

\section{Declarations}

\section{Conflict of Interest}

The authors declare no conflict of interest associated with this manuscript.

\section{Author Contributions}

The roles of the authors were as follows: conception and design (RT, KM, HM); data analysis (TF, TM, YC); data analysis and interpretation (TF, HN, SG); and drafting of the manuscript (RT, TF).

Ethics: Tokai University School of Medicine Ethical Committee approved this clinical project in November, 2021.

\section{References}

1. Witberg, G. et al. Myocarditis after Covid-19 vaccination in a large health care organization. N Engl J Med 385,21322139(2021)

2. Mevorach, D. et al. Myocarditis after BNT162b2 mRNA vaccine against Covid-19 in Israel. N Engl J Med 385,21402149(2021)

3. Truong, D, T. et al. Clinically suspected myocarditis temporally related to COCID-19 vaccination in adolescents and young adults. Circulation, doi:org/10.1161/CIRCULATIONAHA.121.056583(2021)

4. The 74th time health sciences council vaccination vaccine subcommittee meeting associate reaction consideration section and a Fiscal year 2021 25th time Pharmaceutical Affairs and Food Sanitation Council subcommittee meeting medical product of pharmaceutical affairs are sectional investigative committee (It's held jointly.) material of security measures of security measures. 
https://translation.mhlw.go.jp/LUCMHLW/ns/tl.cgi/https://www.mhlw.go.jp/stf/shingi2/0000208910_00036.html? $S L A N G=j a \& T L A N G=e n \& X M O D E=0 \& X P A R A M=q, \& X C H A R S E T=U T F-8 \& X P O R G=, \& X J S I D=0(2021)$

5. Murphy, W, J. \& Longo, D, L. A possible role for anti-idiotype antibodies in SARS-CoV-2 infection and vaccination. N Engl J Med, doi:10.1056/NEJMcibr2113694(2021)

6. Paque, R, E. \& Miller, R. Autoanti-idiotypes exhibit mimicry of myocyte antigens in virus -induced myocarditis. J Virol $65,16-22(1991)$

\section{Figures}

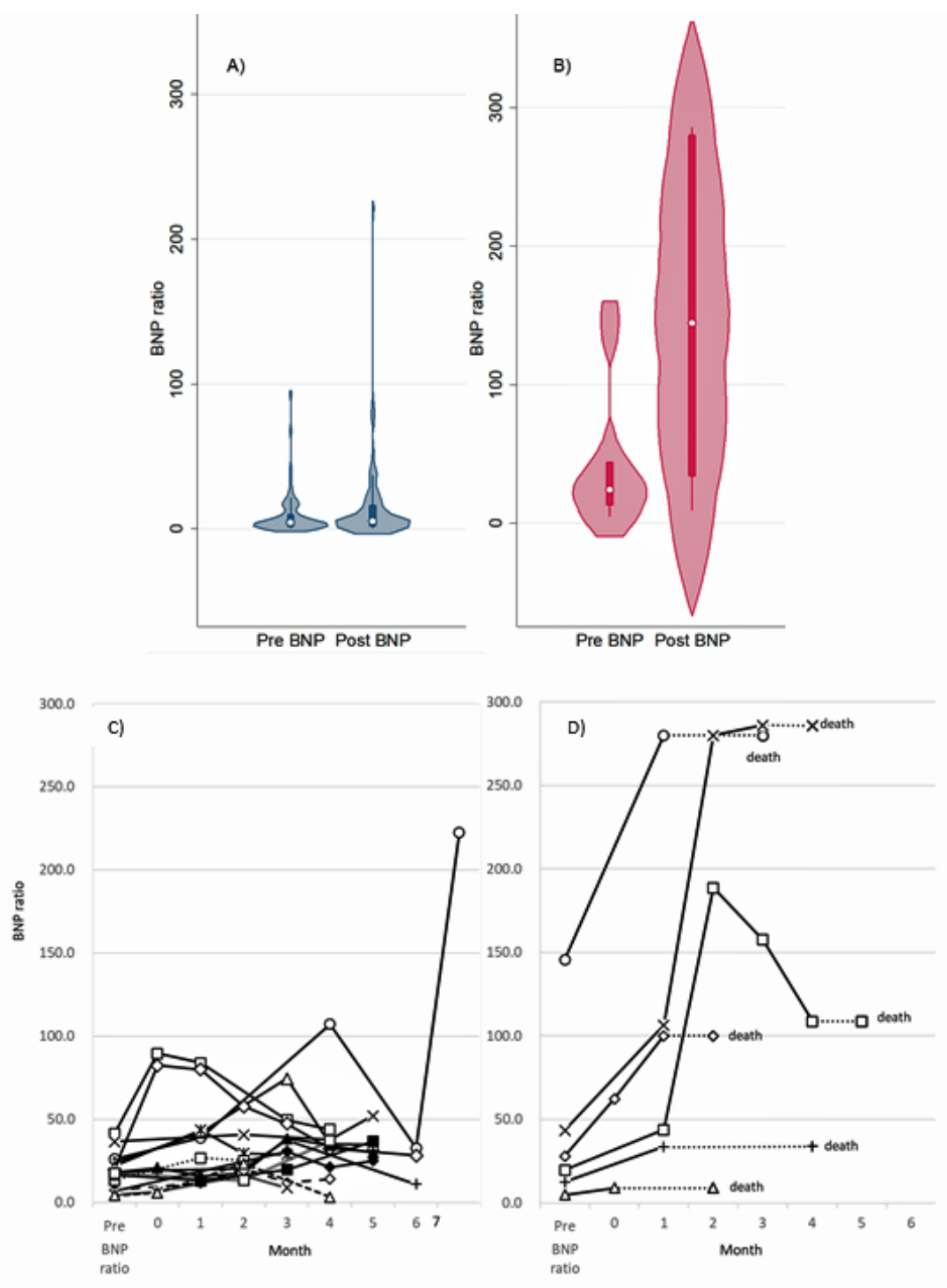

\section{Figure 1}

Figure 1. Distribution of pre- and post-BNP ratios, and Transition of BNP ratios.

The transition of the BNP ratio according to vaccination is displayed as a violin plot. Pre- and post-BNP ratios were 4.2 (1.4-9.5) and $5.2(1.8-16.1)$ in patients who survived (A), and 23.9 (12.6-43.6) and 144.4 (33.8-280) in patients who expired (B),

respectively. 
Transition of BNP ratios in 15 patients who survived (C) and six patients who expired (D). There was a temporary increase in the BNP ratio by $>10$-fold higher than the UNL after vaccination in all 15 patients. Subsequently, eight and seven patients showed partial and little improvement, respectively. Four of the seven patients with little improvement showed sustained increase in the BNP ratio 4-7 months after the vaccination(C). Five of the six patients who expired showed a rapid increase in the BNP ratio prior to death. In the remaining one patient, who expired due to malnutrition as a result of vaccination-induced back pain, we could not determine the post-BNP ratio. Values (i.e., post-BNP levels minus pre-BNP levels) $\geq 10$-fold higher than the UNL denoted significant increment in the BNP ratio(D).

BNP, brain natriuretic peptide; UNL, upper normal limit. 